\title{
From the Sakai-Sugimoto model to the generalized Skyrme model
}

\author{
Lorenzo Bartolini, ${ }^{*}$ Stefano Bolognesi, ${ }^{\dagger}$ and Andrea Proto \\ Department of Physics "E. Fermi", University of Pisa and INFN Sezione di Pisa, \\ Largo Pontecorvo, 3, Ed. C, 56127 Pisa, Italy
}

(Received 28 November 2017; published 31 January 2018)

\begin{abstract}
We derive the generalized Skyrme model as a low-energy effective model of the Sakai-Sugimoto model. The novelty with the past is the presence of the sextic term equal to the topological charge squared. This term appears when the $\omega$ meson, and the tower of states on top of it, are integrated out. We claim that, in the small 't Hooft coupling limit, the instanton is well described by a Skyrmion arising from the low energy effective Lagrangian of the Sakai-Sugimoto model. The sextic term plays a dominant role in this limit. Moreover, when a pion mass term is added, we recover the BPS Skyrme model in the small 't Hooft coupling limit.
\end{abstract}

DOI: 10.1103/PhysRevD.97.014024

\section{INTRODUCTION}

The Sakai-Sugimoto (SS) model is a top-down holographic model of QCD [1,2]. The model, among all the holographic attempts, is one of the closest example to QCD and being a top-down model, it has very few parameters to adjust. The SS model has been successfully applied to reproduce qualitative or semiquantitative properties of QCD both in the mesonic and in the baryonic sector. The mesonic sector contains the pions, which are the Goldstone bosons of the chiral symmetry breaking, plus a tower of massive vector bosons among the $\omega$ meson and the $\rho$ mesons. The Lagrangian, restricted to the pions fields, reproduces exactly the Skyrme model. The model incorporates all the features of the large- $\mathrm{N}$ expansion and in particular, the identification between baryons and Skyrmions-Instantons, which are solitonic objects made out of the mesonic fields [3-5].

In this paper, we want to explore more in detail the relation between the Sakai-Sugimoto model and the effective Skyrme model. Our first task is to find a missing important piece in the pions effective Lagrangian: the sextic term. We just said that, restricting to the pions fields, just by setting the vector mesons to zero, we obtain the familiar Skyrme model with the two terms which are most commonly considered, the quadratic and the quartic one. However, the correct way to

\footnotetext{
*lorenzobartolini89@gmail.com

†stefano.bolognesi@unipi.it

”proto.andrea@yahoo.com
}

Published by the American Physical Society under the terms of the Creative Commons Attribution 4.0 International license. Further distribution of this work must maintain attribution to the author(s) and the published article's title, journal citation, and DOI. Funded by SCOAP. obtain a low-energy effective action is not to set to zero the massive fields by brute force. We should instead integrate them out in order to find any residual interactions to be included in the derivative expansion of the low-energy effective action. We find in fact that a new term is generated by this procedure: a sextic term in a number of derivatives which correspond to the topological charge squared. This is analogous to what happens in the Skyrme model coupled to the $\omega$ vector meson [6], where integrating out the massive meson generates a term proportional to the topological charge squared. Our mechanism is a generalization of the one just cited adapted to the Sakai-Sugimoto model, where the whole tower of vector mesons enters into play. The sextic term has also been previously discussed in [7].

We thus obtain the so-called "generalized Skyrme model", which is the model that contains four terms in the Lagrangian $\mathcal{L}=\mathcal{L}_{0}+\mathcal{L}_{2}+\mathcal{L}_{4}+\mathcal{L}_{6}$, where the subscript corresponds to the number of derivatives of the pion field. $\mathcal{L}_{0}$ is the mass term, or a generic potential, which is generated by an explicit breaking of the chiral symmetry, $\mathcal{L}_{2}$ is the quadratic Dirichlet term, $\mathcal{L}_{4}$ is the quartic Skyrme term, and $\mathcal{L}_{6}$ is the topological charge squared term. The generalized Skyrme model is the most generic effective model with the requirement of having at most two time derivatives. By setting $\mathcal{L}_{6}$ to zero, we reobtain the ordinary massive Skyrme model, which has been studied in depth in the past and has the drawback of predicting classical binding energies too large for nuclei. By setting $\mathcal{L}_{2}$ and $\mathcal{L}_{4}$ to zero, we obtain the so-called BPS Skyrme model $[8,9]$. It is a model in which Skyrmions, in any topological sector, saturate a Bogomolny bound and have a infinite dimensional moduli space corresponding to the volume preserving diffeomorphisms. Starting from the BPS Skyrme model and treating $\mathcal{L}_{2}$ and $\mathcal{L}_{4}$ as perturbations, it is conjectured to have a small nuclear binding energy and to be very close to 
the phenomenology of the liquid-drop model $[9,10]$. The Skyrmion of a topological charge one is spherical and stable against quadrupole deformations in the generalized Skyrme model [11]. In the limit in which $\mathcal{L}_{2}$ and $\mathcal{L}_{4}$ are set to zero, it becomes marginally stable, deformations are allowed and are in fact part of the infinite dimensional group of diffeomorphisms transformations. Of course for physical reasons, we need stability; in this paper, we will always consider $\mathcal{L}_{2}$ and $\mathcal{L}_{4}$ different from zero, although small for small 't Hooft coupling. The interesting aspect of the low-energy effective action of the SS model, for small 't Hooft coupling, is that it falls exactly into this class with the coefficient of all the terms precisely fixed by the UV theory.

In the second part of this paper, we consider the baryonic sector of the SS model and how the generalized Skyrme model may help us to understand it. The picture at a large 't Hoof coupling $\lambda$ is the one that received most of the attention in the past. The whole string theory setup is in fact well approximated by semiclassical computation only in this limit, together with the limit of large $N_{c}$. When the 't Hooft coupling is large, the SS model has instantons solutions, very well approximated by small BPS instantons whose size scales as $\mathcal{O}(1 / \sqrt{\lambda})$, which is much smaller than the bulk radius of curvature. These instantons correspond to the baryons of the dual QCD. They carry the same quantum number of the Skyrmions but they are quite different from the Skyrmions that would be obtained by solving the Skyrme effective Lagrangian in isolation. When instantons are very small, they probe deep into the fifth holographic dimension, and all the tower massive vector mesons enter in their structure.

We here focus our attention on the other, much less studied, limit of small 't Hooft coupling. This limit lies outside the applicability of the top-down string holographic model, but it does make sense if we consider our model as a bottom-up phenomenological model. Moreover, when we calibrate the SS model to the real QCD, we have to choose a particular 't Hooft coupling, which in general is never too large or too small; it falls in the middle between the two limits.

We claim that in the small 't Hooft coupling limit, the instanton becomes very large and eventually can be studied by considering only the generalized Skyrme effective obtained by integrating out all the massive vector meson. So in this limit, the instanton really turns out to be a Skyrmion. In particular, we find that at a very small 't Hooft coupling, only a few terms in the effective Skyrme Lagrangian are important. If pions are massless, they are $\mathcal{L}_{2}+\mathcal{L}_{6}$. If pions are massive, they are instead $\mathcal{L}_{0}+\mathcal{L}_{6}$ thus reproducing the result of the BPS Skyrme model. We thus find the 't Hooft coupling interpolates between two distinct BPS models for baryons: small self-dual instantons for large $\lambda$ and large BPS Skyrmions for small $\lambda$. This is a generalization of what happens in lower dimensions with the baby-Skyrme model [12].
The paper is organized as follows. In Sec. II, we discuss the pion effective Lagrangian obtained by integrating out the massive vector meson. In Sec. III, we consider the baryon at a small 't Hooft coupling, which becomes the Skyrmions of the generalized Skyrme model. We conclude in Sec. IV.

\section{THE SEXTIC TERM DERIVATION FROM THE SS MODEL}

The model, after dimensional reduction to an effective $5 D$ theory, is described by a Yang-Mills/Chern-Simons action [2],

$$
\begin{aligned}
S= & S_{\mathrm{YM}}+S_{\mathrm{CS}} \\
S_{\mathrm{YM}}= & -\kappa \operatorname{tr} \int d^{4} x d z\left[\frac{1}{2} h(z) \mathcal{F}_{\mu \nu}^{2}+k(z) \mathcal{F}_{\mu z}^{2}\right] \\
S_{\mathrm{CS}}= & \frac{N_{c}}{384 \pi^{2}} \epsilon_{\alpha_{1} \alpha_{2} \alpha_{3} \alpha_{4} \alpha_{5}} \int d^{4} x d z \hat{A}_{\alpha_{1}}\left[6 \operatorname{tr}\left(F_{\alpha_{2} \alpha_{3}}^{a} F_{\alpha_{4} \alpha_{5}}^{a}\right)\right. \\
& \left.+2 \operatorname{tr}\left(\hat{F}_{\alpha_{2} \alpha_{3}} \hat{F}_{\alpha_{4} \alpha_{5}}\right)\right],
\end{aligned}
$$

where $\kappa \equiv a N_{c} \lambda$ with $a \equiv\left(216 \pi^{3}\right)^{-1}$, and $k(z)=\left(1+z^{2}\right)$, $h(z)=k(z)^{-1 / 3}$. The field content is given by a $U\left(N_{f}\right)$ connection $\mathcal{A}$,

$$
\mathcal{A}=\hat{A} \frac{\mathbb{1}}{N_{f}}+A^{a} T^{a},
$$

where $T^{a}$ are the generators of $S U\left(N_{f}\right)$ normalized to obey $\operatorname{tr}\left(T^{a} T^{b}\right)=\frac{1}{2} \delta^{a b}$. We will work in the $N_{f}=2$ case, thus accounting for the up and down quarks: in this case, $T^{a}=\frac{\tau^{a}}{2}$.

We can go to the $\mathcal{A}_{z}=0$ gauge with the transformation

$$
\begin{aligned}
\mathcal{A} \rightarrow \mathcal{A}^{g} & =g^{-1} \mathcal{A} g+i g^{-1} d g \\
g & \equiv \exp \left(i \int_{0}^{z} d z^{\prime} \mathcal{A}_{z}\left(x, z^{\prime}\right)\right) .
\end{aligned}
$$

In this gauge, the Chern-Simons Action assumes the form,

$$
\begin{aligned}
S_{\mathrm{CS}}= & \frac{N_{c}}{384 \pi^{2}} \epsilon^{\mu_{1} \alpha_{2} \cdots \alpha_{5}} \int d^{4} x d z \hat{A}_{\mu_{1}}\left[6 \operatorname{tr}\left(F_{\alpha_{2} \alpha_{3}} F_{\alpha_{4} \alpha_{5}}\right)\right. \\
& \left.+2 \operatorname{tr}\left(\hat{F}_{\alpha_{2} \alpha_{3}} \hat{F}_{\alpha_{4} \alpha_{5}}\right)\right] \\
= & \frac{N_{c}}{96 \pi^{2}} \epsilon^{\mu_{1} z \mu_{3} \mu_{4} \mu_{5}} \int d^{4} x d z \hat{A}_{\mu_{1}}\left[6 \operatorname{tr}\left(F_{z \mu_{3}} F_{\mu_{4} \mu_{5}}\right)\right. \\
& \left.+\hat{F}_{z \mu_{3}} \hat{F}_{\mu_{4} \mu_{5}}\right] .
\end{aligned}
$$

In particular, we shall need the following equation of motion for the $U(1) \hat{A}_{\mu}$ field: 


$$
\begin{aligned}
& -\kappa\left(h(z) \partial_{\nu} \hat{F}^{\mu \nu}+\partial_{z}\left(k(z) \hat{F}^{\mu z}\right)\right) \\
& \quad+\frac{N_{c}}{64 \pi^{2}} \epsilon^{\mu \alpha_{1} \cdots \alpha_{4}} \operatorname{tr}\left(\mathcal{F}_{\alpha_{1} \alpha_{2}} \mathcal{F}_{\alpha_{3} \alpha_{4}}\right)=0 .
\end{aligned}
$$

In the gauge adopted, we know that the following fields expansion holds [1]:

$$
\mathcal{A}_{\mu}=\mathcal{U}^{-1} \partial_{\mu} \mathcal{U} \psi_{+}+\sum_{n=1}^{\infty} B_{\mu}^{(n)}(x) \psi_{n}(z),
$$

where the first term accounts for the pion component $\psi_{+} \equiv-\frac{i}{2}\left(1+\frac{2}{\pi} \arctan z\right)$, while the second sum includes all vector and axial-vector mesons. We employ an Abelian ansatz for the vector meson part, and moreover, we assume that it factorizes as follows:

$$
\mathcal{A}_{\mu}=\left\{\begin{array}{l}
\hat{A}_{\mu}=B_{\mu}(x) \chi(z) \\
A_{\mu}=\mathcal{U}^{-1} \partial_{\mu} \mathcal{U} \psi_{+}(z) .
\end{array}\right.
$$

With this ansatz, the field strength becomes

$$
\begin{aligned}
& F_{\mu \nu}=\left[R_{\mu}, R_{\nu}\right] \psi_{+}\left(i \psi_{+}-1\right) \\
& F_{z \mu}=R_{\mu} \psi_{+}^{\prime} \\
& \hat{F}_{\mu \nu}=f_{\mu \nu} \chi \\
& \hat{F}_{z \mu}=B_{\mu} \chi^{\prime},
\end{aligned}
$$

where we have defined $R_{\mu}=\mathcal{U}^{-1} \partial_{\mu} \mathcal{U}$ and $f_{\mu \nu} \equiv \partial_{\mu} B_{\nu}-$ $\partial_{\nu} B_{\mu}$.

Plugging (2.8) and (2.7) into (2.5), we obtain the following differential equation:

$$
\begin{aligned}
& 2 \kappa z B_{\mu} \chi^{\prime}+\kappa\left(1+z^{2}\right) B_{\mu} \chi^{\prime \prime}+\kappa h(z) \partial_{\nu} f^{\nu \mu} \chi \\
& +\frac{N_{c}}{16 \pi^{2}} \epsilon^{\mu z \mu_{1} \mu_{2} \mu_{3}}\left\{\operatorname{tr}\left(R_{\mu_{1}}\left[R_{\mu_{2}}, R_{\mu_{3}}\right] \psi_{+} \psi_{+}^{\prime}\left(i \psi_{+}-1\right)\right)\right. \\
& \left.\quad+\frac{1}{2}\left(B_{\mu_{1}} f_{\mu_{2} \mu_{3}} \chi^{\prime} \chi\right)\right\}=0 .
\end{aligned}
$$

Solving this, we get $B_{\mu}$ as a function of $R_{\mu}$. We want to obtain a low energy $4 D$ effective action so we want to keep the term with lowest number of four-dimensional derivatives. For this reason, we omit both terms in (2.9) that depend on $f_{\mu \nu}$. Keeping the remaining terms, we obtain two equations, one for $B_{\mu}$ and the other for the profile function $\chi(z)$ in the holographic direction,

$$
\begin{array}{r}
2 z \chi^{\prime}+k(z) \chi^{\prime \prime}=\frac{N_{c}}{16 \kappa \pi^{2}} \psi_{+} \psi_{+}^{\prime}\left(i \psi_{+}-1\right) \\
B_{\mu}(x)=-\epsilon_{\mu}{ }^{z \nu_{1} \nu_{2} \nu_{3}} \operatorname{tr}\left(R_{\nu_{1}}\left[R_{\nu_{2}}, R_{\nu_{3}}\right]\right) .
\end{array}
$$

This justifies the factorization of the ansatz (2.7) for the vector meson part. The function $\chi(z)$ can be obtained in an analytical form by solving its equation of motion with boundary conditions $\chi( \pm \infty)=0$,

$\chi=-\frac{N_{c}}{64 \pi^{3} \kappa}\left(\frac{5 \pi^{2}}{48}-\frac{1}{2} \arctan ^{2}(z)+\frac{1}{3 \pi^{2}} \arctan ^{4}(z)\right)$.

It is trivial to check that with this solution for $B_{\mu}$, the neglected terms in (2.9) are all of a higher $L^{-1}$ order than the ones we kept.

We now plug the ansatz (2.7) into the action and use Eqs. (2.10), (2.11) to express everything in terms of $R_{\mu}$. For the Chern-Simons term, we obtain

$$
\begin{aligned}
S_{\mathrm{CS}}= & \frac{N_{c}}{96 \pi^{2}} \epsilon^{\mu_{1} z \mu_{3} \mu_{4} \mu_{5}} \int d^{4} x d z B_{\mu_{1}} \chi\left[6 \operatorname{tr}\left(R_{\mu_{3}}\left[R_{\mu_{4}}, R_{\mu_{5}}\right]\right)\right. \\
& \left.\times \psi_{+} \psi_{+}^{\prime}\left(i \psi_{+}-1\right)+B_{\mu_{3}} f_{\mu_{4} \mu_{5}} \chi^{\prime} \chi\right] .
\end{aligned}
$$

Let us first concentrate on the first term. If we make use of the equation for $B_{\mu}(x)$ and use the fact that a commutator antisymmetrized via the totally antisymmetric tensor amounts to 2 times the product, we find

$$
\begin{aligned}
S_{\mathrm{CS}}^{(1)}= & -\frac{N_{c}}{4 \pi^{2}} \epsilon^{z \mu_{1} \mu_{3} \mu_{4} \mu_{5}} \epsilon^{z \mu_{1} \nu_{3} \nu_{4} \nu_{5}} \\
& \times \int d^{4} x \operatorname{tr}\left(R_{\mu_{3}} R_{\mu_{4}} R_{\mu_{5}}\right) \operatorname{tr}\left(R_{\nu_{3}} R_{\nu_{4}} R_{\nu_{5}}\right) \\
& \times \int d z \chi \psi_{+} \psi_{+}^{\prime}\left(i \psi_{+}-1\right) \\
= & -\frac{N_{c}}{4 \pi^{2}} \int d^{4} x\left(\epsilon^{z \mu_{1} \nu_{3} \nu_{4} \nu_{5}} \operatorname{tr}\left(R_{\nu_{3}} R_{\nu_{4}} R_{\nu_{5}}\right)\right)^{2} \\
& \times \int d z \chi \psi_{+} \psi_{+}^{\prime}\left(i \psi_{+}-1\right) .
\end{aligned}
$$

Performing the integral and using the solution for $\chi(z)$ (2.12), we obtain the following sextic term:

$$
S_{6}^{\mathrm{CS}}=\frac{51 N_{c}}{4480 \lambda} \int d^{4} x\left(\epsilon^{z \mu_{1} \nu_{3} \nu_{4} \nu_{5}} \operatorname{tr}\left(R_{\nu_{3}} R_{\nu_{4}} R_{\nu_{5}}\right)\right)^{2} .
$$

The second term of (2.13) vanishes because it can be reduced to a boundary term, and $\chi(z)$ vanishes for $z \rightarrow \pm \infty$,

$S_{\mathrm{CS}}^{(2)} \propto \int_{-\infty}^{+\infty} d z \chi^{\prime} \chi^{2}=\frac{1}{3} \int_{-\infty}^{+\infty} d z \partial_{z}\left(\chi^{3}\right)=\frac{1}{3}\left[\chi^{3}\right]_{-\infty}^{+\infty}=0$.

Another contribution to the sextic term arises from the Abelian part of the Yang-Mills action. Of the two terms, the one without derivatives in $z$ turns out to be of order $L^{-8}$; hence, we neglect it, as we have done with its contribution to the equation of motion. Then, the only contribution to a sextic term comes from the action, 


$$
\begin{aligned}
S_{6}^{\mathrm{YM}} & =-\frac{\kappa}{2} \int d z d^{4} x k(z) \hat{F}_{z \mu}^{2} \\
& =-\frac{51 N_{c}}{8960 \lambda} \int d^{4} x\left[\epsilon^{\mu z \nu_{1} \nu_{2} \nu_{3}} \operatorname{tr}\left(R_{\nu_{1}} R_{\nu_{2}} R_{\nu_{3}}\right)\right]^{2} .
\end{aligned}
$$

The full effective sextic term is then given by $S_{6}=$ $S_{6}^{\mathrm{CS}}+S_{6}^{\mathrm{YM}}$,

$$
S_{6}=\frac{51 N_{c}}{8960 \lambda} \int d^{4} x\left[\epsilon^{\mu z \nu_{1} \nu_{2} \nu_{3}} \operatorname{tr}\left(R_{\nu_{1}} R_{\nu_{2}} R_{\nu_{3}}\right)\right]^{2} .
$$

We now want to investigate which are the vector mesons we are integrating out of our action to obtain the sextic term. In the Sakai-Sugimoto model, the whole tower of vector and axial-vector mesons is included in the field $\mathcal{A}$ as noted in the field expansion (2.6): the functions $\psi_{n}(z)$ correspond for each value of $n$ to a certain meson. The defining equation for the functions $\psi_{n}$ is given by

$$
-h(z)^{-1} \partial_{z}\left(k(z) \partial_{z} \psi_{n}\right)=\lambda_{n} \psi_{n},
$$

where the eigenvalues $\lambda_{n}$ determine the mass of the mesons. We can assume that the functions $\psi_{n}$ are even or odd functions of $z$, since (2.19) is invariant under $z \rightarrow-z$. If we order the values $\lambda_{n}$ to be increasing with $n$, then the associated eigenfunctions are of alternate parity, starting with an even $\psi_{1}$. From (2.6), we can see that modes that are even (odd) in $z$ correspond to vector (axial) mesons: the lightest vector meson is then to be associated to the function $\psi_{1}$.

The functions are normalized via the orthonormality condition

$$
\int d z h(z) \psi_{n} \psi_{m}=\delta_{n m}
$$

So we normalize the function $\chi(z)$ in the same way,

$$
\begin{gathered}
|\chi|^{2}=\int d z h(z) \chi^{2}(z), \\
\chi^{(\mathrm{norm})}(z) \equiv \frac{\chi(z)}{|\chi|} .
\end{gathered}
$$

In order to extract the meson content of our solution, we then project the function $\chi(z)$ onto the set of eigenfunctions via the product

$$
a_{n} \equiv \int d z h(z) \chi^{(\text {norm })} \psi_{n}
$$

The functions $\psi_{n}$ can be numerically obtained by a shooting method and, since the function $\chi(z)$ is even, we can perform just the projections involving $\psi_{2 n-1}$, signifying that we are integrating out only the vector meson tower. Our results for the squared coefficients $a_{n}^{2}$ are the following:

$a_{1}^{2}=0.988, \quad a_{3}^{2}=0.0115, \quad a_{5}^{2}=0.00029$.

We thus see that most of the contribution comes from the $\omega$ meson, which is the one associated to the function $\psi_{1}$.

In the SS model, a mass term for the quarks can be introduced via the Aharony-Kutasov action [13],

$$
S_{\mathrm{AK}}=c \int d^{4} x \operatorname{tr} \mathcal{P}\left[M\left(e^{-i \int d z \mathcal{A}_{z}^{(\mathrm{bg})}}-\mathbb{1}\right)+\text { c.c. }\right] .
$$

This term breaks the gauge invariance: if we fix a gauge, then we must account for the gauge variation of this term. The field $\mathcal{A}_{z}^{(\mathrm{bg})}$ in this action should be regarded as having the form it had before moving to the gauge $\mathcal{A}_{z}=0$ (bg stands for "before gauge"). We know that the pathordered exponential of the $\mathcal{A}_{z}$ field is to be identified with the pion matrix $\mathcal{U}$ as follows:

$$
\mathcal{P} e^{-i \int d z \mathcal{A}_{z}^{(\mathrm{bg})}}=\mathcal{U}(x)
$$

Now we take the quark mass matrix to be diagonal, and the masses of the up and down quark to be degenerate and equal to $m$, so we end up with

$$
S_{\mathrm{AK}}=m c \int d^{4} x \operatorname{tr}[(\mathcal{U}-\mathbb{1})+\text { c.c. }] .
$$

We can now adopt the usual decomposition of the field $\mathcal{U}$

$$
\mathcal{U} \equiv \sigma+i \vec{\pi} \cdot \vec{\tau},
$$

together with the unitary constraint

$$
\sigma^{2}+|\vec{\pi}|^{2}=1
$$

The trace, the part valued in $S U(2)$, vanishes, while the complex conjugate accounts for a factor of 2 , so we end up with the following potential:

$$
S_{0}=4 m c \int d^{4} x(\sigma-1) .
$$

Remembering that the non-Abelian part of the YangMills action produces the quadratic and the quartic term of a Skyrme model (after trivial integrations of the holographic coordinate), we can now write down explicitly the full Lagrangian

$$
S=S_{6}+S_{4}+S_{2}+S_{0}
$$

with 


$$
\begin{aligned}
& S_{6}=\frac{51 N_{c}}{8960 \lambda} \int d^{4} x\left[\epsilon^{\mu z \nu_{1} \nu_{2} \nu_{3}} \operatorname{tr}\left(R_{\nu_{1}} R_{\nu_{2}} R_{\nu_{3}}\right)\right]^{2} \\
& S_{4}=a \lambda N_{c} \int d^{4} x \operatorname{tr}\left(\left[R_{\mu}, R_{\nu}\right]^{2}\right) \\
& S_{2}=\frac{\lambda N_{c}}{216 \pi^{4}} \int d^{4} x \operatorname{tr}\left(R_{\mu} R^{\mu}\right) \\
& S_{0}=4 m c \int d^{4} x(\sigma-1),
\end{aligned}
$$

where $a \equiv 1.17 \times 10^{-5}$ and $m c$ fixed by the Gell-MannOakes-Renner relation $4 m c=f_{\pi}^{2} m_{\pi}^{2}$. We are thus left with a generalized Skyrme model provided with the usual pion mass potential $S_{0}$.

Setting to zero the $S U(2)$ valued vector mesons as we have done in the ansatz (2.7) does not give the full effective Lagrangian: other terms are generated if we integrate out all the vector meson. Let us comment on this choice by turning on the $\rho$ meson (the same considerations hold for all the vectors). In this case, the new field content reads

$$
\mathcal{A}_{\mu}=\left\{\begin{array}{l}
\hat{A}_{\mu}=B_{\mu}(x) \chi(z) \\
A_{\mu}=R_{\mu} \psi_{+}(z)+B_{\mu}^{(1)}(x) \psi_{1}(z) .
\end{array}\right.
$$

Now we now look at the equation for the field $A_{\mu}^{a}$,

$$
\begin{gathered}
-\kappa\left[h(z)\left(D_{\nu} F^{\mu \nu}\right)^{a}+\partial_{z}\left(k(z) F^{\mu z}\right)^{a}\right] \\
+\frac{N_{c}}{64 \pi^{2}} \epsilon^{\mu \alpha_{1} \cdots \alpha_{4}} F_{\alpha_{1} \alpha_{2}}^{a} \hat{F}_{\alpha_{3} \alpha_{4}}=0 .
\end{gathered}
$$

We can again think that in an effective field theory approach, every field will be of some order $L^{-k}$, with the pion field being the leading term (with the lowest value of $k$ ): if the new field $B_{\mu}^{(1)}$ is of the same order of the Abelian vector meson $B_{\mu}$, then it is possible for it to generate quartic and sextic order effective potentials for the pion field. However, if this is the case, all the leading order terms in the equation of motion would come from the Yang-Mills action, resulting in a $\rho$ meson field of order $B^{(1)} \psi_{1} \sim \mathcal{O}\left(\lambda^{0}\right)$, while we have $B_{\mu} \chi \sim \mathcal{O}\left(\lambda^{-1}\right)$. We expect the factorization proposed to be valid in the small $\lambda$ regime, so that the $\rho$ meson contribution to the sextic potential will be suppressed as $\lambda^{2}$ and not included in our analysis, while we will argue that the quartic potential becomes negligible anyway in both the massive and massless models as $\lambda$ becomes small.

\section{BARYONS AS SKYRMIONS}

We start evaluating the static action where we set every time derivative to zero,

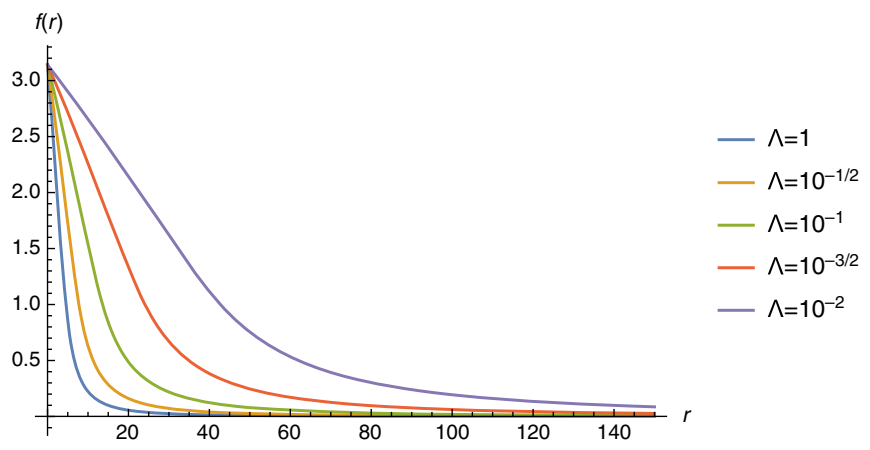

FIG. 1. Skyrmion profile for decreasing values of $\Lambda$. As can be seen, the size of the soliton solution increases as $\Lambda$ becomes small.

$$
\begin{aligned}
S_{\text {static }}= & \frac{\lambda N_{c}}{216 \pi^{4}} \int d^{4} x \operatorname{tr}\left(R_{i} R_{i}\right)+a \lambda N_{c} \int d^{4} x \operatorname{tr}\left(\left[R_{i}, R_{j}\right]^{2}\right) \\
& -\frac{51 N_{c}}{8960 \lambda} \int d^{4} x\left[\epsilon^{i j k} \operatorname{tr}\left(R_{i} R_{j} R_{k}\right)\right]^{2}
\end{aligned}
$$

Note that the minus sign in the sextic term comes from $\eta_{00}=-1$ used to contract $\epsilon^{\mu_{1} \mu_{2} \mu_{3} \mu_{4}}$. Here, we make the reasonable assumption that the solution is spherical symmetric. For triaxial scaling deformations, this can be proven to be stable, see, for example [11]. We thus employ the usual Skyrme model $B=1$ hedgehog ansatz,

$$
\mathcal{U}(x)=e^{i f(r) \hat{x} \cdot \vec{\tau}}
$$

The specific form of $f(r)$ will be the one which minimizes the static energy. Remembering that $S=-\int d t E$, we find

$$
\begin{aligned}
E= & \frac{\lambda N_{c}}{27 \pi^{3}} \int d r\left(r^{2} f^{\prime 2}+2 \sin ^{2} f\right) \\
& +64 \pi a \lambda N_{c} \int d r\left(\frac{\sin ^{4} f}{r^{2}}+2 \sin ^{2} f f^{\prime 2}\right) \\
& +\frac{459 \pi N_{c}}{140 \lambda} \int d r \frac{f^{\prime 2}}{r^{2}} \sin ^{4} f .
\end{aligned}
$$

From this expression, we can derive the Euler-Lagrange equation for the function $f(r)$,

$$
\begin{aligned}
& \sin 2 f\left\{f^{\prime 2}\left(b+\frac{\alpha}{\Lambda^{2}} \frac{\sin ^{2} f}{r^{2}}\right)-b \frac{\sin ^{2} f}{r}-1\right\} \\
& +2 f^{\prime}\left\{r-\frac{\alpha}{\Lambda^{2}} \frac{\sin ^{4} f}{r^{3}}\right\}+f^{\prime \prime}\left\{r^{2}+2 b \sin ^{2} f+\frac{\alpha}{\Lambda^{2}} \frac{\sin ^{4} f}{r^{2}}\right\}
\end{aligned}
$$$$
=0 \text {, }
$$

where we have introduced the following parameters:

$\Lambda \equiv \frac{8 \lambda}{27 \pi}, \quad b=1728 \pi^{4} a \simeq 1.97, \quad \alpha \simeq 76.701$. 


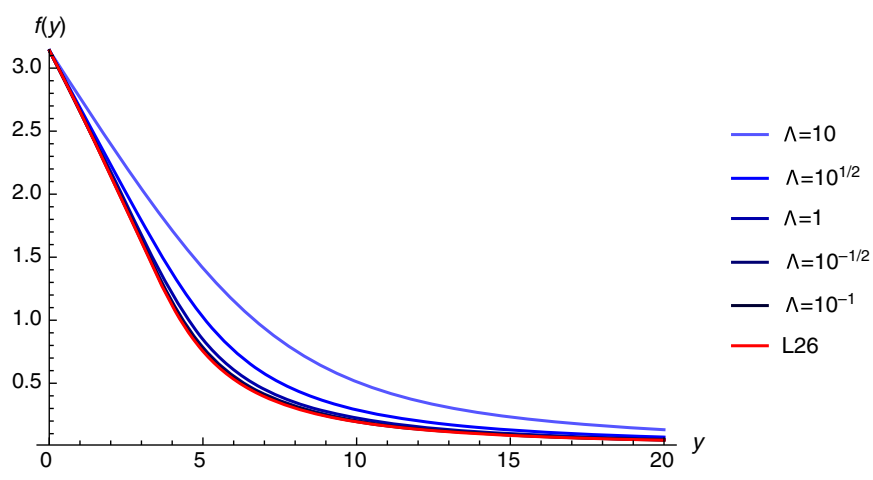

FIG. 2. Skyrmion's profile functions rescaled to the same size. In red, we have plotted the rescaled solution of (3.8), in shades of blue, the ones of the full massless model.

The equation has to be solved with the usual boundary values $f(0)=\pi$ and $f(\infty)=0$ : this can be achieved numerically by a shooting method, with results shown in Fig. 1 for various values of the $\Lambda$ parameter.

Following Derrick's theorem [14], we expect the size of the soliton to scale as $\Lambda^{1 / 2}$ in the small $\Lambda$ limit: in fact, rescaling the coordinates as $x^{i} \rightarrow R x^{i}$, we can see from (3.1) that the various contributions to the static energy scale like

$$
E(R)=\Lambda R E_{2}+\Lambda \frac{E_{4}}{R}+\frac{E_{6}}{\Lambda R^{3}} .
$$

Imposing $\frac{d E(R)}{d R}=0$ and the small $\Lambda$ limit, we find that $R \sim \Lambda^{-1 / 2}$, so that in this regime the energy becomes

$$
\left.E(R)\right|_{R=\Lambda^{-1 / 2}}=\frac{1}{R}\left(E_{2}+E_{6}\right)=\Lambda^{1 / 2}\left(E_{2}+E_{6}\right),
$$

and thus, the model should approach a Skyrme model involving only the kinetic term and the sextic term $\left(\mathcal{L}_{26}\right.$ model $)$.

To explicitly check that this is indeed the case, we rescale the solutions of the massless model adopting a new coordinate $y \equiv r \Lambda^{1 / 2}$ for various values of $\Lambda$, and we plot the profile functions $f(y)$ together with the one of the $\mathcal{L}_{26}$ model, which can be obtained by solving

$$
\begin{aligned}
& \sin 2 f\left\{f^{\prime 2} \frac{\alpha}{\Lambda^{2}} \frac{\sin ^{2} f}{r^{2}}-1\right\}+2 f^{\prime}\left\{r-\frac{\alpha}{\Lambda^{2}} \frac{\sin ^{4} f}{r^{3}}\right\} \\
& +f^{\prime \prime}\left\{r^{2}+\frac{\alpha}{\Lambda^{2}} \frac{\sin ^{4} f}{r^{2}}\right\}=0 .
\end{aligned}
$$

The results of the numerical solution are plotted in Fig. 2: it is manifest that as $\Lambda$ decreases, the quartic term rapidly becomes negligible, and the solution of the $\mathcal{L}_{246}$ model approaches that of the $\mathcal{L}_{26}$ one in the large $\Lambda$ limit; otherwise, we should expect the soliton size to become independent of $\Lambda$ as can be seen again from (3.6): choosing

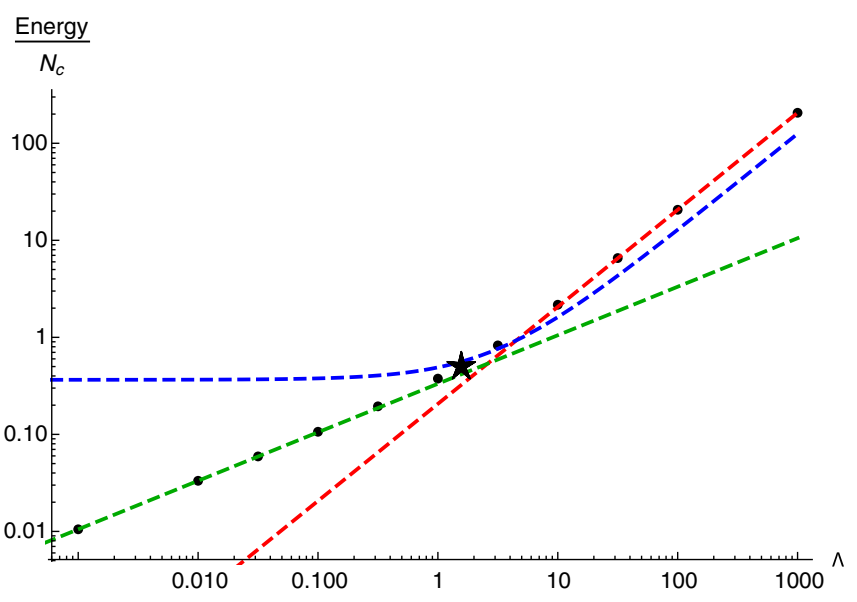

FIG. 3. Energy for a range of values of $\Lambda$ in the massless model. Black dots are the energy obtained with the model developed. The dashed red and green lines correspond, respectively, to fitting linear and square root relations, as are expected to develop in the large and small $\Lambda$ regions. The dashed blue line represents the energy of the BPST instanton, which becomes the correct description of the baryon at large $\Lambda$ [4]. The black star corresponds to the energy computed at the phenomenological value of $\Lambda=1.568$ : as can be seen, this value lies just in between the two regimes of large and small 't Hooft coupling.

$R \sim 1$, we can again minimize the energy, except that this time, the analysis is valid in the large $\Lambda$ region.

Finally, we check these considerations by explicitly computing the total energy for a wide range of values of $\Lambda$, with results shown in the plot in Fig. 3. As can be seen, at small $\Lambda$, the energy dependence on this parameter correctly approaches the square root one, as predicted by the Derrick's theorem.

It is now interesting to check what happens to the Skyrmion when we consider the full massive model, that is the generalized Skyrme model with a pion mass potential, in both the generic and small $\Lambda$ regime.

The new potential $S_{0}$ modifies the equation of motion for the profile function $f(r)$, which now reads

$$
\begin{aligned}
& \sin 2 f\left\{f^{\prime 2}\left(b+\frac{\alpha}{\Lambda^{2}} \frac{\sin ^{2} f}{r^{2}}\right)-b \frac{\sin ^{2} f}{r}-1\right\} \\
& +2 f^{\prime}\left\{r-\frac{\alpha}{\Lambda^{2}} \frac{\sin ^{4} f}{r^{3}}\right\}+f^{\prime \prime}\left\{r^{2}+2 b \sin ^{2} f+\frac{\alpha}{\Lambda^{2}} \frac{\sin ^{4} f}{r^{2}}\right\} \\
& \quad-m_{\pi}^{2} r^{2} \sin f=0,
\end{aligned}
$$

where we have used the explicit form of $f_{\pi}$ given by the holographic model, which can be read from the coefficient of $S_{2}$ in (2.32), and once written in terms of the new parameter (3.5), it amounts to

$$
f_{\pi}^{2}=\frac{\Lambda N_{c}}{16 \pi^{3}} .
$$

As before, we can solve (3.9) via a shooting method with the same boundary condition $[f(0)=\pi, f(\infty)=0]$ 


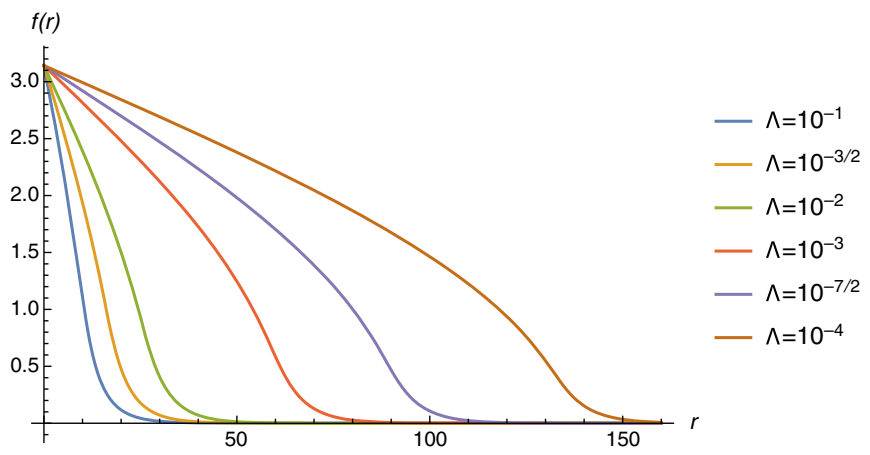

FIG. 4. Skyrmion's profile functions in the generalized model with a pion mass potential.

to have the $B=1$ soliton. With regards to the mass of the pion, we use the phenomenological value of $m_{\pi^{0}}$, measured in units of $M_{K K}$,

$$
m_{\pi} \equiv \frac{m_{\pi^{0}}^{\mathrm{ph}}}{M_{K K}} \simeq \frac{135 \mathrm{MeV}}{949 \mathrm{MeV}} \simeq 0.142 .
$$

The plot in Fig. 4 shows the different shape that the function assumes in the small $\Lambda$ regime, which can be traced back to the model effectively becoming a BPS Skyrme model with only the mass and sextic potentials $\left(\mathcal{L}_{06}\right)$.

We can understand this behavior in the same way as before, observing that this time the soliton size scales as $\Lambda^{-1 / 3}$ in the small $\Lambda$ regime: after the rescaling, the energy contributions scale like

$$
E(R)=\Lambda R E_{2}+\Lambda \frac{E_{4}}{R}+\frac{E_{6}}{\Lambda R^{3}}+\Lambda R^{3} E_{0}
$$

Minimizing this energy leads again to two possibilities for the size $R$ of the soliton: $R \sim \Lambda^{-1 / 3}$ or $R \sim 1$. The latter is again the correct scaling in the large $\Lambda$ regime and yields to the usual linear dependence of the total energy on $\Lambda$, while the former is appropriate in the small $\Lambda$ limit and, as can be easily checked from (3.12), makes the energy independent of the $\Lambda$ parameter in this limit,

$$
\begin{gathered}
\left.E(R)\right|_{R=\Lambda^{-1 / 3}} \stackrel{\Lambda \rightarrow 0}{\longrightarrow} \frac{E_{6}}{\Lambda R^{3}}+\Lambda R^{3} E_{0}=E_{6}+E_{0} \\
\left.E(R)\right|_{R=1} \stackrel{\Lambda \rightarrow \infty}{\longrightarrow} \Lambda\left(E_{2}+E_{4}+E_{0}\right) .
\end{gathered}
$$

Both the behaviors are explicitly shown in Fig. 5.

The behaviors at large $\Lambda$ are extrapolations reported to compare with the correct description of the baryons in that regime, that is a BPS configuration localized deep in the holographic direction. As can be seen, in both the massive and the massless models, the values of the static energy obtained by this extrapolation show the same power-law dependence on $\Lambda$ as the ones obtained from the actual

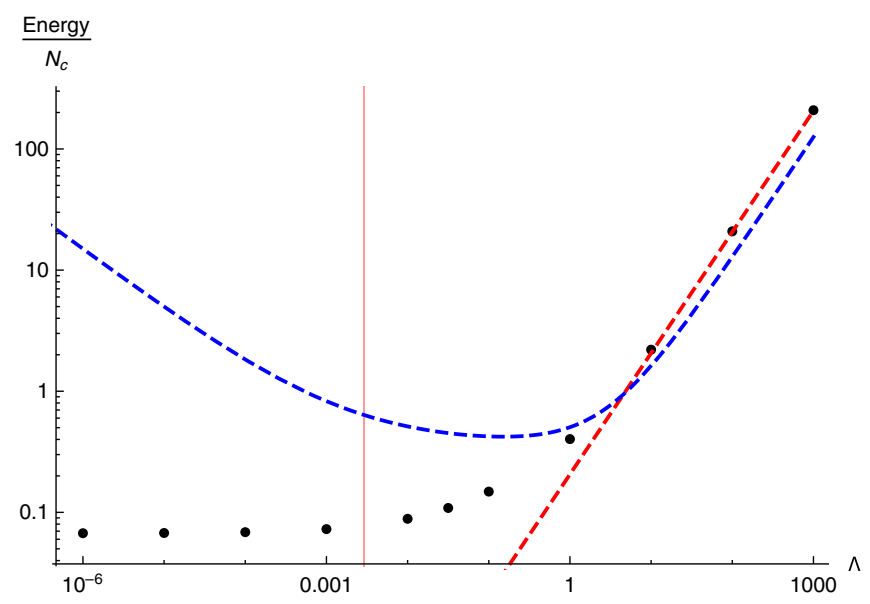

FIG. 5. Energy for a range of values of $\Lambda$. The dashed red line corresponds to a fitting linear relation, as it is expected to develop in the large $\Lambda$ region. The vertical red line corresponds to the value satisfying $\Lambda=R^{-3}=m_{\pi}^{3}$, which is expected to be the scale at which the change in behavior happens. The blue dashed line again represents the energy of the BPST configuration, which describes the baryon at large $\Lambda$, with the addition of the shift in mass computed in [15] due to the quark mass term.

holographic description, but differ from these by an excess in their normalization.

The reduction to the BPS $\mathcal{L}_{06}$ model is even more manifest if we again plot the rescaled profile functions against the analytical compacton solution of such a model: following [16], if the Lagrangian reads

$$
\mathcal{L}=\frac{\gamma^{2}}{24^{2}}\left[\epsilon^{\mu \nu_{1} \nu_{2} \nu_{3}} \operatorname{tr}\left(R_{\nu_{1}} R_{\nu_{2}} R_{\nu_{3}}\right)\right]^{2}-\mu^{2}(1-\sigma)
$$

adopting the hedgehog ansatz (3.2), the compacton solution is given by

$$
f(r)= \begin{cases}2 \arccos (A r) & \text { for } r \in\left[0, A^{-1}\right] \\ 0 & \text { for } r \geq A^{-1}\end{cases}
$$

with the inverse length scale being $A=\sqrt[3]{\frac{3 \sqrt{2} \mu}{4 \gamma}}$.

We can map the parameters $\gamma$ and $\mu$ to the ones of our model just by looking at the action coefficients of $S_{0}$ and $S_{6}$, resulting in the identification,

$$
\gamma^{2}=\frac{\alpha N_{c}}{8 \Lambda \pi^{3}}, \quad \mu^{2}=\frac{\Lambda N_{c} m_{\pi}^{2}}{16 \pi^{3}},
$$

so that the size $A^{-1}$ of the compacton becomes $A^{-1}=$ $\sqrt[3]{\frac{4 \sqrt{\alpha}}{\Lambda m_{\pi}}}$. Note that, as expected, the size of the compacton scales as $\Lambda^{-1 / 3}$. In Fig. 6, we have plotted the solutions of the full equation (3.9), rescaled to the same unitary size via the redefinition $y=A r$, for a wide range of values of $\Lambda$. As can be seen, the profile functions $f(r)$ approach the analytical one for decreasing $\Lambda$, but not as fast as in the massless case examined before: to reach a good match, we 


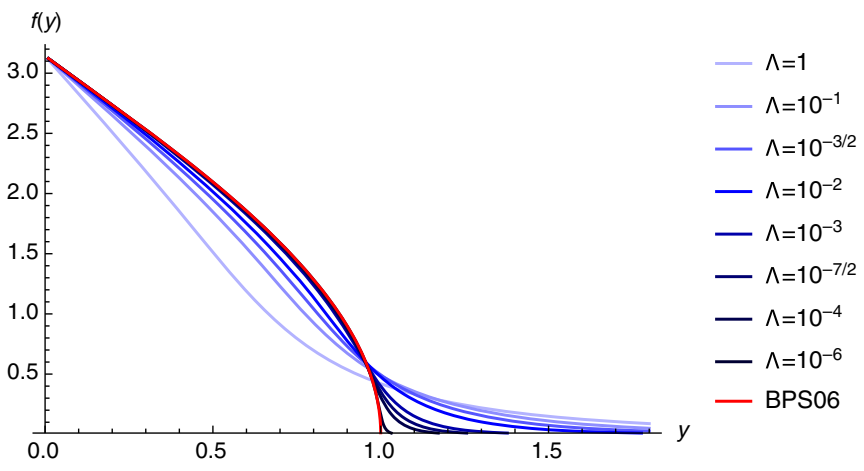

FIG. 6. Skyrmion's profile functions rescaled to the same size. In red, we have plotted the rescaled analytical solution of the BPS model $\mathcal{L}_{06}$, in shades of blue, the ones of the full massive equation (3.9).

have to push $\Lambda$ way down to values of at least $10^{-6}$, while the massless solution was already in great agreement with the one of the $\mathcal{L}_{26}$ model for $\Lambda=10^{-1}$.

\section{CONCLUSION}

The Sakai-Sugimoto model at low energies may be written as a derivative expansion in the pion fields. In particular, we computed the sextic term, which is generated by an integration over the $\omega$ meson and the whole mesonic tower on top of it. This term becomes particularly important in the small 't Hooft coupling limit. We also conjectured that the Instanton baryon, as the 't Hooft coupling becomes small, is well approximated by the Skyrmion computed from the low energy model, which is a generalized version of the Skyrme model. When the pions are strictly massless, the dominant terms are $\mathcal{L}_{2}+\mathcal{L}_{6}$. If pions are massive, we recover the BPS Skyrme model $\mathcal{L}_{0}+\mathcal{L}_{6}$.

Phenomenological calibration of the SS model requires a choice of the 't Hooft coupling that in general is neither too big or too small. Up to now, only the very large 't Hooft coupling region has been analytically solved by the fact that instantons become almost self-dual. We showed here that there is another region in the parameter space where great simplification occurs for the baryons, that of small $\lambda$. This may help in understanding better the solution in the intermediate regime which, so far, can be accessed only with numerical methods [17].

In proximity of the BPS Skyrme model, the quantization of the Skyrmion may produce large deviations from the spherical symmetric state. In other words, the validity of the rigid rotor approximation may become questionable in this regime. These corrections should be carefully studied when aiming to reproduce the nuclear states phenomenology from an almost-BPS Skyrme model.

Skyrme models in general have always been plagued by the problem of predicting classical binding energies too large. Quantum effects can help reducing the binding energies, but in general is always better to start from something already close to the real value at the classical level. In this respect, it is quite interesting to have a model that is never too far from a BPS substructure. The SS model with the massive deformation has exactly this feature, for a big 't Hooft coupling $\lambda$ becomes the self-dual Yang-Mills plus small corrections, while a small $\lambda$ becomes the BPS Skyrme model plus small corrections. It remains to be seen if this feature can help in reducing, and how much, the classical binding energy also in the intermediate region of the parameter space.

\section{ACKNOWLEDGMENTS}

We thank C. Adam, I. Basile, P. Sutcliffe, and A. Wereszczynski, for useful discussions. The present work is supported by the INFN special research project grant GAST ("Gauge and String Theories").
[1] T. Sakai and S. Sugimoto, Low energy hadron physics in holographic QCD, Prog. Theor. Phys. 113, 843 (2005).

[2] T. Sakai and S. Sugimoto, More on a holographic dual of QCD, Prog. Theor. Phys. 114, 1083 (2005).

[3] D. K. Hong, M. Rho, H. U. Yee, and P. Yi, Chiral dynamics of baryons from string theory, Phys. Rev. D 76, 061901 (2007).

[4] H. Hata, T. Sakai, S. Sugimoto, and S. Yamato, Baryons from instantons in holographic QCD, Prog. Theor. Phys. 117, 1157 (2007).

[5] K. Hashimoto, T. Sakai, and S. Sugimoto, Holographic baryons: Static properties and form factors from gauge/ string duality, Prog. Theor. Phys. 120, 1093 (2008).
[6] A. Jackson, A. D. Jackson, A. S. Goldhaber, G. E. Brown, and L. C. Castillejo, A modified Skyrmion, Phys. Lett. 154B, 101 (1985).

[7] P. Colangelo, J. J. Sanz-Cillero, and F. Zuo, Holography, chiral Lagrangian and form factor relations, J. High Energy Phys. 11 (2012) 012.

[8] C. Adam, J. Sanchez-Guillen, and A. Wereszczynski, A BPS Skyrme model and baryons at large $N_{c}$, Phys. Rev. D 82, 085015 (2010).

[9] C. Adam, C. Naya, J. Sanchez-Guillen, and A. Wereszczynski, Bogomol'nyi-Prasad-Sommerfield Skyrme Model and Nuclear Binding Energies, Phys. Rev. Lett. 111, 232501 (2013). 
[10] M. Gillard, D. Harland, and M. Speight, Skyrmions with low binding energies, Nucl. Phys. B895, 272 (2015).

[11] C. Hajduk and B. Schwesinger, Excitation of deformed Skyrmions, Nucl. Phys. A453, 620 (1986).

[12] S. Bolognesi and W. Zakrzewski, Baby Skyrme model, near-BPS approximations and supersymmetric extensions, Phys. Rev. D 91, 045034 (2015).

[13] O. Aharony and D. Kutasov, Holographic duals of long open strings, Phys. Rev. D 78, 026005 (2008).
[14] G. H. Derrick, Comments on nonlinear wave equations as models for elementary particles, J. Math. Phys. (N.Y.) 5, 1252 (1964).

[15] K. Hashimoto, T. Hirayama, and D. K. Hong, Quark mass dependence of hadron spectrum in holographic QCD, Phys. Rev. D 81, 045016 (2010).

[16] C. Adam, J. Sanchez-Guillen, and A. Wereszczynski, A BPS Skyrme model, J. Phys. Conf. Ser. 284, 012006 (2011).

[17] S. Bolognesi and P. Sutcliffe, The Sakai-Sugimoto soliton, J. High Energy Phys. 01 (2014) 078. 